\title{
$\sqrt{6}$

\section{Métodos de valoración ambiental aplicados a la regeneración y reutilización de aguas residuales en agricultura}

\section{Environmental Assessment Methods Applied to the Regeneration and Reuse of Wastewater in Agriculture}

\author{
Universitat Politècnica de Catalunya. Castelldefels, Barcelona, España. oscar. alfranca@upc.edu
}

\begin{abstract}
Resumen - El análisis económico y financiero de los proyectos de sistemas de regeneración y reutilización de aguas residuales se ha concentrado frecuentemente en los costes y beneficios privados, tanto por las dificultades metodológicas relacionadas con la estimación de las externalidades, como por la disponibilidad de datos necesarios para su valoración. La consideración de los costes y beneficios ambientales permite una mejora sustancial en el diseño y gestión de los sistemas de regeneración y reutilización de aguas residuales, ya sean públicos o privados. Como consecuencia, también se produce una mejora en la gestión de las inversiones en estos sistemas de tratamiento. El objetivo de este trabajo es presentar unos fundamentos básicos para los principales métodos de valoración ambiental. Y muy especialmente, cuando estos son aplicados en actividades relacionadas con el uso de aguas residuales en agricultura.

Abstract - The economic and financial analysis of wastewater reuse systems projects has laid emphasis on the issues of private costs and benefits, both because of methodological difficulties related to estimating existing externalities and the lack of available data needed for its measurement. Taking full account of the environmental costs and benefits allows for a substantial improvement in the design and management of public and private wastewater reuse systems. Consequently, an improvement is also obtained in the management of investments in the treatment systems. The main objective of this paper is to present the essential theoretical foundations for the main environmental valuation methods, and most particularly when they are applied to wastewater use in agriculture.
\end{abstract}

\begin{abstract}
Palabras clave: métodos de valoración ambiental, aguas residuales, agricultura, preferencias reveladas, preferencias declaradas Keywords: environmental valuation methods, wastewater, agriculture, revealed preferences, stated preferences
\end{abstract}


La utilización de aguas residuales en agricultura ha recibido una atención creciente en los últimos tiempos. Los motivos principales son la escasez de agua para riego y el interés por disminuir la presencia de sustancias contaminantes en el medio ambiente. En los proyectos relacionados con el riego agrario, resulta imprescindible aplicar unos métodos específicos de valoración ambiental, puesto que las metodologías convencionales no son válidas para estimar todas sus consecuencias económicas y ambientales. Por ejemplo, el método de análisis coste-beneficio requiere de la monetización de los efectos ambientales relacionados, tales como la instalación de una planta desaladora y sus posibles efectos sobre el suelo marino. Habitualmente estos bienes y servicios ambientales no pueden asignarse mediante un mercado y carecen de precio. Todavía más compleja es la valoración de bienes que no presentan un uso claro, pero sí un valor por su propia existencia, como podría ser un tipo de alga específica o un paisaje rural. El objetivo de este trabajo es presentar una descripción de los principales métodos de valoración ambiental y, muy especialmente, algunas bases sobre su aplicación en el uso de aguas residuales en agricultura. El artículo comienza con una breve presentación de la literatura más reciente sobre este tema. Posteriormente, se describen algunos conceptos económicos esenciales en el análisis de proyectos sobre agua regenerada. La sección tercera trata sobre la colaboración público-privada en los servicios de saneamiento y abastecimiento de agua. Con posterioridad, se presentan algunos fundamentos sobre los principales métodos de valoración. Para acabar, se discuten algunos problemas metodológicos y se ofrecen unas conclusiones.

\section{Agua Regenerada y AGricultura}

La reutilización de agua para riego es una actividad cada vez más frecuente en agricultura, tanto en zonas rurales, como urbanas y periurbanas. Las aguas regeneradas y reutilizadas pueden contener diferentes substancias, en función de la fuente en que fueron obtenidas y de su nivel de tratamiento ${ }^{1}$. Con frecuencia el precio de las aguas residuales tratadas es menor al de las aguas convencionales, lo que supone un incentivo usar aguas residuales por los regantes ${ }^{2}$.

Por ejemplo, algunos de los componentes principales de las aguas residuales y regeneradas como nitrógeno, fósforo y potasio pueden utilizarse como nutrientes para plantas. Entre los posibles elementos nocivos se hallan algunas sales, metales y metaloides, pesticidas, componentes tóxicos orgánicos y microcontaminantes ${ }^{3}$.

Existe un gran número de estudios en los que se estiman los beneficios para la actividad agraria relacionados con el agua regenerada 4 . Estas investigaciones descartan posibles consecuencias sanitarias cuando se cumplen unos protocolos establecidos.

1 Pescod, 1992. Qadir et al, 2007a. Jiménez y Asano, 2008. Keralta et al. 2010. Qadir et al. 2015.

2 Hurliman y McKay, 2007.

3 Siemens et al. 2008. Simmons et al. 2010.

4 Ver por ejemplo Hamilton et al. 2007. Qadir et al. 2007b. Scott et al. 2004a. Scott et al. 2004b. Scheierling et al. 2011.
Sin embargo, el problema podría ser diferente cuando el agua se obtiene de manera informal, sin consideración al guna por las condiciones sanitarias ${ }^{5}$ puesto que la concentración de componentes nocivos (por encima de unos límites permitidos) podría originar problemas en la salud humana ${ }^{6}$.

\section{Externalidades y bienes Públicos EN LOS PROYECTOS DE AGUA REGENERADA}

Una hipótesis fundamental en economía es que los cambios en los precios de los bienes modifican el bienestar de consumidores y de productores. Si bien la cuantía de estos cambios puede estimarse mediante el excedente del consumidor y el excedente del productor, en el caso de los bienes de no-mercado las variaciones en el bienestar originadas por modificaciones en los precios y cantidades consumidas, deben medirse mediante los cambios en la curva que indica la disposición a pagar por parte de los consumidores (puesto que no existe un precio para estos bienes).

Las curvas que representan la disposición marginal a pagar los bienes públicos y los bienes de no-mercado son imprescindibles para expresar la demanda de algunos servicios ambientales agrarios, tales como formación del paisaje rural o la mejora en la biodiversidad de una comarca. Sin embargo, esta disposición podría estimarse mediante la observación directa de las transacciones relacionadas, puesto que no existe un mercado para los bienes públicos y ambientales. Por tanto, la búsqueda de métodos alternativos que permitan estimar la demanda de estos bienes, resulta ineludible.

El valor económico de un recurso natural 0 ambiental puede calcularse como la suma de los valores presentes del bien, una vez descontados los flujos de sus servicios asociados. Los bienes ambientales inciden en el bienestar humano de manera diversa. Una primera etapa en la valoración de estos bienes consiste en determinar el rango de los impactos esperados, es decir, las características del bien que le otorgan su valor y que deberían recogerse mediante técnicas de valoración ambiental. La suma de los diferentes valores parciales permite cal cular el Valor Económico Total, que representa el conjunto de efectos sobre el bienestar humano.

Dado que algunos de estos fluj os y servicios no pueden comprarse 0 venderse en un mercado (y por tanto no tienen precio) el valor económico de este recurso natural podría ser muy diferente al de su valor comercial o residencial. Un precio de mercado sería muy difícil que pudiera recoger el valor de los servicios asociados a la biodiversidad de la zona o su función como elemento sustancial en la formación del paisaje rural.

Una hipótesis principal en la estimación de los costes y beneficios del agua regenerada es que cualquier intervención pública puede modificar el flujo de servicios asociados y por tanto provocar cambios en el valor presente de este bien. Es decir, que cualquier alteración en el flujo de bienes y servicios presentaría su contrapartida en el valor económico del bien. Algunos de estos fluj os presentan vinculaciones con el mercado y podría generarse

5 Drechsel et al. 2010. Raschid-Sally y Jayakody, 2008.

6 WHO, 2006. 


\section{Métodos de valoración ambiental aplicados a la regeneración}

y reutilización de aguas residuales en agricultura

una respuesta a estos cambios. Sin embargo, muchos de estos flujos no están propiamente determinados por el mercado, sino por otros factores con características de bien público.

Las características que definen a un bien público son dos: la no exclusión y la no rivalidad. Por la capacidad de exclusión entendemos que existe la posibilidad de limitar el acceso de un individuo a este bien (ya sea para su consumo o para utilizarlo como factor de producción). La condición de rivalidad se refiere a la imposibilidad de que varios individuos puedan consumir la misma unidad del mismo bien. Si se satisfacen estas dos condiciones, un sistema de mercado descentralizado podría no alcanzar una asignación óptima. Es decir, que tal vez sería necesaria la intervención pública en su gestión (que requeriría de una mejora en los flujos de información asociados).

Las externalidades relacionadas con las aguas residuales aparecen cuando empresas 0 individuos generan costes 0 beneficios sobre otros agentes sociales y estos costes o beneficios no son compensados. Para garantizar la gestión eficiente de cualquier proyecto de agua regenerada, la cuantificación de estas posibles consecuencias resulta imprescindible. En un proyecto de reutilización, por ejemplo, deberían considerarse las siguientes externalidades positivas: aumento en la disponibilidad de agua, disminución en el uso de fertilizantes y disminución en la sobre explotación de acuíferos, entre otras. Las externalidades negativas se relacionan esencialmente con los riesgos químicos y biológicos.

Las aguas residuales presentan con frecuencia las características de bien público, puesto que buena parte de la sociedad las consume y se beneficia de ellas sin necesidad de pagar por su disponibilidad. Así, por ejemplo, resulta difícil la exclusión del consumo individual de un humedal artificial que se ha construido utilizando aguas residuales y que ha mejorado las condiciones de biodiversidad de la zona y sus valores paisajísticos. La naturaleza no rival de los beneficios relacionados con el consumo de aguas residuales, así como su dificultad de exclusión, justifican en la mayoría de los casos la necesidad de su regulación pública. Pese a las características de bien público de las aguas regeneradas, existen beneficios relacionados con su utilización o consumo que justifican las decisiones privadas. Es decir, que habría agentes privados dispuestos a invertir tiempo y esfuerzo (así como su posible coste de oportunidad) para utilizarlas como input en un proceso productivo. Esta perspectiva de bien privado podría referirse tanto a las aguas residuales tratadas como a las que no lo han sido. Por ejemplo, agencias de agua en Australia, Israel y los Estados Unidos pueden vender aguas tratadas a posibles agricultores y propietarios de campos de golf, (ya sea directamente o mediante un programa de recarga de acuíferos) y obtener beneficios mediante su uso como agua de riego?.

Un aspecto fundamental en la asignación de aguas residuales tratadas es que, a causa de la presencia de externalidades, bienes comunales, y de las características de bien público de algunos de estos bienes (por ejemplo, en una zona de humedales) el funcionamiento del mercado no permite siempre la asignación

\footnotetext{
7 Mills et al. 2004. Van Roon, 2007.
}

de las aguas residuales a los usos más valiosos, a unos precios que incorporen todos sus valores sociales. Por los mismos motivos, tampoco podría darse un proceso de revelación de preferencias al precio que permitiera reflejar todos los valores sociales del agua regenerada. Es decir, que en un mercado sin restricciones, el precio de las aguas residuales podría ser ineficiente. Un aspecto sustancial es que, con frecuencia, los consumidores están dispuestos a pagar un precio superior al de mercado, antes que renunciar a alguno de los servicios ambientales asociados.

El cálculo de los costes y beneficios ambientales cada vez resulta más habitual en los proyectos hidráulicos. Sin embargo, su elevada complejidad metodológica, así como la dificultad para contrastar empíricamente las valoraciones, determina que la aplicación de estos métodos presente con frecuencia muchas dificultades. En el caso de las aguas residuales, queda patente la importancia de los efectos ambientales en la Directiva Marco del Agua (DMA). La DMA representa un paradigma en la aproximación a la planificación hidrológica y entre sus conceptos fundamentales se encuentra el de recuperación de los costes. Este principio implica que en el cálculo de los costes y beneficios relacionados con la reutilización del agua deberían considerarse no solamente los costes financieros, sino también los costes y beneficios ambientales.

La valoración económica de los bienes ambientales supone una información muy relevante en los proyectos de reutilización. El valor total de un bien se define como la suma de su valor de uso y de no-uso. Los valores de uso son los que derivan de la utilización del bien, y se ven afectados por cualquier cambio en el entorno. El valor de no-uso se refiere a la disposición a pagar por unos recursos que no se utilizan, ni piensan utilizarse en el futuro. El principal de estos valores es el valor de existencia, que refleja el bienestar que unas personas obtienen del simple conocimiento de que un bien existe. Por su propia naturaleza, los valores de no-uso son al mismo tiempo valores no comerciales.

El principio de recuperación de costes en la DMA comporta que en los proyectos de aguas regeneradas se consideren no solamente los costes financieros, sino también los costes y beneficios ambientales. Con frecuencia no existe un mercado para estos bienes, y por tanto no pueden asignarse mediante un precio. Es decir, que para reconocer su valor monetario sería necesario aplicar unas técnicas de valoración ambiental. La valoración económica de los bienes ambientales resulta fundamental en el proceso de toma de decisiones de la gestión pública.

En la gestión de las empresas de aguas residuales, los dos objetivos principales son la recuperación de los costes de inversión y el suministro de agua con un grado de calidad aceptable para los usuarios. La recuperación de los costes mediante la venta del agua tratada para riego agrario suele ser un objetivo incumplido, que solamente se satisface gracias a la intervención pública. En los países en desarrollo resulta extraño que los agricultores paguen por el agua (ya sea esta superficial o de pozo) más allá de unos costes de extracción. Por otra parte, no es extraño que las aguas tratadas o canalizadas reciban algún tipo de subsidio.

Aunque no es muy habitual que el precio del agua para riego permita recuperar todos los costes relacionados con la instala- 
ción de la planta, sí que existe un cierto margen para determinar un precio que permita garantizar la recuperación de los costes iniciales (cuando no incluso la obtención de un beneficio, en el caso de las industrias). En general, la utilización más rentable de las aguas residuales se relaciona con el consumo humano. En este caso, el agua puede atravesar por diversos procesos como microfiltración, ósmosis inversa o irradiación mediante rayos UVA (o alguna tecnología que permita un grado de desinfección similar). Estos procesos permiten crear agua con un nivel de calidad semejante al agua de consumo común, por lo que su precio también sería superior, y podría utilizarse para usos domésticos 0 ambientales.

El uso de los flujos de agua tratada descargada en el medio ambiente desde plantas de tratamiento municipales constituye una estrategia fundamental para el ahorro en los cuerpos naturales de agua. Los flujos de aguas tratadas (si se descargan sin haber recibido el tratamiento necesario) podrían ocasionar efectos negativos sobre los cuerpos receptores. La viabilidad de una planta de reutilización depende de circunstancias locales que determinan su balance de costes y beneficios. En la mayoría de las ocasiones, el mayor beneficio radica en la disponibilidad de agua para consumo humano, que es posible obtener a partir de agua de riego, agua para uso industrial, o bien agua utilizada con fines ambientales. En resumen, el análisis de un proyecto de planta de tratamiento debería realizarse desde una perspectiva municipal o regional, y aplicando un análisis coste-beneficio, en el que se consideren no solamente los efectos privados sino también los sociales y ambientales.

\section{El ANÁlisis COSTE-BENEFiCIO}

El análisis coste-beneficio es un método para la toma de decisiones y suele aplicarse en la selección de proyectos para la instalación de plantas de tratamiento de aguas residuales. En la selección se consideran no solamente los proyectos con un coste menor (análisis coste-eficiencia) sino aquellos en que la diferencia entre ingresos y costes es máxima.

Desde la década de los años 50, cuando se desarrollaron las técnicas más básicas del análisis coste-beneficio, se han producido unos cambios muy significativos en la naturaleza de los problemas analizados, así como en los instrumentos analíticos utilizados. Estos métodos son los que permiten el análisis y la valoración de bienes ambientales relacionados con los proyectos de aguas residuales.

El método de análisis Coste-Beneficio se desarrolló inicialmente para calcular el Valor Actualizado Neto (VAN), en proyectos públicos de inversión, y muy especialmente en inversiones relacionadas con el agua, que utilizaban los factores de producción con la finalidad de obtener un conjunto de productos finales (como agua potable, energía hidroeléctrica y agua de riego). La mayoría de estos bienes se comercializan en el mercado, de modo que la estimación de su valor monetario es inmediata.

El análisis coste-beneficio constituye un instrumento fundamental para la toma de decisiones relacionada con el agua regenerada y permite contrastar si la decisión de reutilizar el agua es la correcta en comparación con otros usos alternativos del agua. En este método, la calidad del agua residual podría considerarse como un input más en el proceso productivo y devenir un fundamento esencial en la gestión sostenible del agua.

$\mathrm{Si}$ existen diferencias sustanciales entre el valor obtenido mediante el uso del agua y el que podría obtenerse a través de su mejor utilización posible, estaría justificada la consideración del coste de oportunidad como un coste del proyecto (si no se escoge el uso más rentable). Algunos problemas esenciales en la gestión del agua son las condiciones relacionadas con su calidad y el decrecimiento de su disponibilidad. Estas consecuencias podrían comportar algunos efectos muy relevantes como la intrusión salina, la contaminación por agroquímicos, el deterioro de humedales y estuarios, la destrucción de hábitats o la disminución de la biodiversidad.

\section{Costes de infraestructuras del agua}

Las condiciones competitivas de mercado no se dan habitualmente en el sector de las aguas residuales, a causa en buena parte de los elevados costes de infraestructura, que podrían inducir el suministro de unos servicios a precios ineficientes (excesivamente altos) desde una perspectiva privada.

En los proyectos de agua regenerada, los costes de inversión en la infraestructura inicial (creación, expansión o rehabilitación de la red de tuberías) suelen ser muy elevados. Habitualmente estos costes se refieren a inversiones que suponen un gasto de construcción único e irreversible, y resulta complejo evaluar su disponibilidad. Dado que se trata de inversiones que suponen un gasto de construcción único e irreversible, suelen considerarse como un monopolio natural.

Un monopolio natural es un monopolio en una industria que es la única capaz de producir a un coste medio mínimo en el largo plazo, con una diferencia muy substancial respecto al resto de competidores. La consecuencia principal es que, como se trata de un mercado con una sola empresa, los precios podrían ser excesivamente elevados, la producción ineficiente y reducirse la calidad tanto en la producción final como en los servicios de distribución. Esta situación suele darse en industrias en las que los costes de capital son muy elevados (y fundamentales para el desarrollo del negocio) y las economías de escala son substanciales en relación al tamaño del mercado. Es decir, se trata de un mercado en el que existen grandes barreras de entrada.

Muchos autores han propuesto el uso de aguas residuales en diferentes escalas y otros han descrito métodos y guías para promover el uso seguro de las aguas residuales. ${ }^{8}$. De hecho, la provisión de un servicio como el suministro de aguas residuales por parte de las empresas requiere de un balance entre los intereses públicos y privados, que pueden presentar intereses divergentes. Así, la vertiente pública suele asociarse a la responsabilidad y la obligación de proporcionar servicios a los habitantes y a los contribuyentes de una zona. Por otra parte, si el servicio lo ofrece una empresa privada, debería asegurarse una compensación, que

8 Ensink y Van der Hoek, 2009. Molinos et al. 2012. 


\section{Métodos de valoración ambiental aplicados a la regeneración}

y reutilización de aguas residuales en agricultura

podría ser imprescindible para cubrir los riesgos asociados con el mantenimiento de las infraestructuras necesarias.

\section{El Partenariado Público-Privado (PPP) \\ EN LA GESTIÓN DE LOS SERVICIOS DE SANEAMIENTO Y ABASTECIMIENTO DE AGUA}

Durante gran parte del siglo XX, los sistemas de abastecimiento de agua en la mayoría de las áreas urbanas de los países industrializados fueron de propiedad pública. Así, los servicios de abastecimiento y saneamiento se desarrollaban con criterios en los que primaba la capacidad de pago de los usuarios y se gestionaban con bajas expectativas de beneficio privado. Es decir, bajo un principio de equidad social. De lo anterior, el agua se consideraba como un bien público y su acceso como una condición previa para la participación política y social.

El partenariado público-privado es un tipo de organización que se ha aplicado especialmente en la provisión de agua para el consumo público. Otros sectores, como el sanitario, también utilizan con frecuencia esta forma societaria a causa del elevado gasto inicial en infraestructuras. Existe una variación considerable entre la inversión de capital y la transferencia de tecnología, la gestión y los recursos operativos que intervienen en cada sector. Las asociaciones PPP se han utilizado en una variedad de formas en los sectores de infraestructura, incluyendo la inversión de capital en proyectos muy especializados, tales como aeropuertos, carreteras, puentes y los proyectos de generación de energía, así como en proyectos de educación y servicios de salud. El problema más importante relacionado con el uso de agua regenerada para la producción agraria está relacionado con la salud. Así, el objetivo primordial en el tratamiento de las aguas residuales radica en sus condiciones sanitarias y en los posibles efectos sobre la salud pública que podría conllevar su consumo.

La incapacidad del sector privado para ampliar la cobertura de los servicios en barrios de rentas bajas, con independencia de su capacidad de pago, fue determinante para que la mayoría de gobiernos municipales asumieran la gestión pública de estos servicios. El resultado fue que gran parte de los sistemas de suministro de agua acabaron gestionados por los poderes públicos, con el objetivo fundamental de alcanzar su universalización. En los casos en los que se mantuvo la gestión privada (como en Inglaterra y Francia) se procedió a una regulación muy detallada. Por ejemplo, en las empresas inglesas los dividendos estaban limitados y cualquier excedente debía ser reinvertido en el sistema.

En este nuevo escenario, el sector público asumió la responsabilidad de gestionar y promover el suministro de agua potable en la mayor parte del mundo industrializado. En este sistema, las empresas ofertantes acaban configurando un monopolio natural en el que no son aplicables las reglas del mercado. En la nueva regulación, a este servicio se le exigen condiciones de acceso universal y salud pública, además de convertirse en un sector que requiere de fuertes inversiones para la ampliación de las coberturas a zonas de bajo poder adquisitivo, con tasas de retorno privadas muy reducidas.
Pese a que se ha producido un aumento considerable de los acuerdos PPP en el sector del agua y las aguas residuales durante las dos últimas décadas, todavía existen particularidades en la utilización de esta forma de organización en el sector del agua regenerada. Esencialmente, el principal elemento diferenciador es que el agua constituye un elemento primordial para la vida humana. Por este motivo, al contrario de otros servicios públicos, como la electricidad, comunicaciones o aeropuertos, los individuos no disponen de alternativas a la utilización de este servicio, lo que comporta la obligación de garantizar este servicio a todos los posibles usuarios (tanto si pueden pagar su utilización como si no es así). Dado que este servicio suele considerarse como básico, suele haber una discusión sobre si debería haber un beneficio o no asociado con su funcionamiento. En el caso del agua regenerada, esta dificultad se manifiesta en el bajo beneficio del servicio si se compara, por ejemplo, con sectores como las telecomunicaciones.

En la posición de dominio monopolístico que suelen presentar las empresas PPP en el sector de las aguas residuales, se manifiesta la ventaja de un dominio previo para mercados y servicios. Resulta habitual que, en el largo plazo, el control en la gestión y oferta de estas empresas, comporte algún tipo de regulación pública de control.

\section{Métodos de ESTIMACIÓN DEL VALOR AMBIENTAL APLICADOS A LAS AGUAS RESIDUALES}

La sobreexplotación de los recursos hídricos suele considerarse como uno de los principales efectos que derivan, entre otros, de la inexistencia de un mercado, puesto que para este tipo de bienes no resulta posible el ajuste entre oferta y demanda. Los instrumentos reguladores disponibles permitirían fijar un precio, basado en algún tipo de normativa pública (de especial importancia en las aguas residuales). En este caso, resulta esencial la disponibilidad de métodos de valoración que permitan considerar el valor de las posibles externalidades relacionadas con los servicios generados.

La valoración de las aguas residuales se realiza mediante un conj unto de técnicas que permiten cuantificar el valor económico de los bienes ambientales. Su objetivo principal es la expresión en unidades monetarias de los cambios en el bienestar de las personas, asociados con las transformaciones relacionadas con el uso o la existencia de las aguas residuales. Bajo la hipótesis de que el bienestar de las personas se origina mediante la satisfacción de sus preferencias, la medida del bienestar podría expresarse a través de su disposición a pagar (o a ser compensado) por el cambio sobre un estado inicial. Existen dos grandes grupos de métodos para la valoración de bienes y servicios que se fundamentan en las preferencias de los individuos: los métodos de preferencias reveladas y los métodos de preferencias declaradas.

La principal distinción entre estos métodos de valoración radica en la fuente de los datos. Así, los datos pueden obtenerse mediante observaciones directas de las personas afectadas, 0 bien a través de un conjunto de respuestas a preguntas hipotéticas. En el primer caso, la información se obtiene por la revelación 
de las preferencias (método de preferencias observadas o reveladas). Y en el segundo, las preferencias se declaran (método de preferencias declaradas o hipotéticas).

Los métodos de valoración se fundamentan en un conjunto de hipótesis básicas:

* Los cambios ambientales deben incidir en la utilidad de los individuos de forma significativa (mayor que cero) en términos monetarios.

* El valor total de un cambio en las condiciones ambientales es igual a la suma de los cambios individuales.

* Los diferentes cambios en el medio ambiente pueden ser comparables una vez calculados en unidades monetarias.

* Otros bienes ambientales del mismo valor podrían actuar como bienes sustitutivos, sin pérdida de bienestar.

Los valores de no-uso se corresponden con aquellos beneficios que no necesitan deuna relación directa entre los consumidores y el bien. Es decir, que no es necesaria la utilización del bien (ya sea directa o indirecta) para obtener su valor. Estos valores suelen conocerse como el valor de existencia, y derivan sencillamente del conocimiento de que este bien existe, ya sea por sí mismo o para el uso que pueda hacerse de él.

\section{Métodos de valoración del agua Regenerada. ConCEPTOS PRINCIPALES}

Las externalidades generadas por el agua, así como sus características de bien público, permiten explicar los fallos en los mecanismos de mercado y la necesidad de utilizar métodos de valoración ambiental para gestionar la toma de decisiones, tanto públicas como privadas.

La valoración económica de los recursos ambientales se fundamenta en que estos recursos presentan impactos diversos sobre la utilidad de los individuos. En el caso de las aguas tratadas, la valoración monetaria de estos efectos podría considerarse como un indicador de los cambios en la utilidad de las personas, directamente relacionado con la disponibilidad del agua. Esta hipótesis se sustenta en que los consumidores deben escoger entre bienes diversos y en la utilidad que puede obtenerse mediante su utilización.

La economía ha desarrollado un conjunto de técnicas para estimar el valor de los bienes de no-mercado. Bajo el supuesto de que el bienestar de las personas se origina mediante la satisfacción de sus preferencias, la medida del bienestar podría expresarse a través de su disposición al pago o de la compensación ante un cambio en la situación o estado inicial.

Existen básicamente dos métodos para estimar los cambios en la demanda 0 en la oferta de los bienes de no-mercado. Los métodos basados en las preferencias reveladas y los métodos basados en las preferencias declaradas. El primero se fundamenta en la estimación del valor a partir de la observación de bienes muy semejantes. El segundo, a partir de las respuestas a unas preguntas hipotéticas.

\section{Métodos de PREFERENCIAS REVELADAS}

Los cambios en los precios de los bienes modifican el bienestar de consumidores y de productores. La magnitud de estos cambios puede calcularse mediante el excedente del consumidor y el excedente del productor, respectivamente. En el caso de bienes de no-mercado, los cambios en el bienestar generados por las modificaciones en las cantidades consumidas (no existe precio para estos bienes) pueden medirse mediante los cambios en la curva, que indicaría la disposición a pagar por parte de los consumidores. Las curvas que representan la disposición marginal a pagar son necesarias para caracterizar bienes públicos y bienes de no-mercado, tales como los servicios del medio ambiente (por ejemplo, el mantenimiento de unos humedales artificiales) pero no pueden estimarse a partir de la observación directa de las transacciones en estos bienes. Dada la ausencia de mercado para bienes públicos y ambientales, es necesario emplear métodos alternativos para calcular su demanda.

Los métodos de preferencias reveladas se basan en las relaciones que se establecen entre los bienes o servicios ambientales objeto de valoración y los que se adquieren en el mercado. Las personas revelan en su comportamiento con respecto al bien privado el valor que realmente le otorgan al bien ambiental. Debido a la relación entre bien privado y bien ambiental que implica los métodos de preferencias declaradas, la aplicación de estos métodos se circunscribe a la estimación de los valores de uso. Otra limitación de los métodos de preferencias declaradas es que solo pueden medir la valoración de los bienes a posteriori, una vez consumidos, por lo que no permiten estimar valores para niveles de calidad que todavía no han sido experimentados. En estos casos podrían extrapolarse los valores obtenidos en situaciones ex- ante como en proyectos de características parecidas o bien utilizar métodos de preferencias declaradas. Los principales métodos de preferencias reveladas son el método del coste de viaje, costes evitados y el método de los precios hedónicos.

La principal finalidad del método del coste de viaje consiste en estimar el valor recreativo del recurso considerado (por ejemplo, un humedal) mediante un indicador como el gasto que los visitantes deben realizar para llegar a visitar este lugar. Gracias al conocimiento de este gasto resulta posible estimar una función de demanda, que podría indicar la disponibilidad al pago por día de visita. Es decir, que el método se basa en el reconocimiento de que los gastos necesarios en el viaje son un componente principal en el coste de visita del recurso. Este supuesto provoca que puedan producirse variaciones en los resultados según cual sea la muestra de visitantes utilizada.

El método del coste de viaje se modeliza con frecuencia como una demanda agregada (por ejemplo, la correspondiente a todos los visitantes de un humedal) o bien como demandas individuales, que después pueden agregarse. La función de demanda individual (representada, por ejemplo, mediante el número de visitas) suele especificarse como una función dependiente de un conjunto de características socioeconómicas y ambientales, entre las que se incluye el precio de entrada al lugar. Este se con- 
sidera un buen indicador puesto que habitualmente el precio se mantiene constante en el tiempo.

Los métodos de los costes evitados, coste de reposición 0 coste de sustitución son métodos utilizados para estimar el valor de los servicios que ofrecen los ecosistemas 9 . El cálculo se basa en cualquiera de los costes necesarios para evitar daños por los servicios perdidos, y muy especialmente con el costo de reemplazar los servicios del ecosistema, o el costo de la prestación de servicios sustitutivos. Así, por ejemplo, los primeros estudios sobre los efectos de la contaminación del agua sobre los productores se basaban en el método de la función de daños ambientales. Esta aproximación se fundamenta en la estimación de una función daño-dosis que relaciona las medidas de polución con la medida física del daño (por ejemplo, mediante el inventario de materiales expuestos al riesgo de contaminación). Para calcular el valor total de la posible externalidad, una vez calculado el daño individual, este se multiplica por el conjunto de unidades afectadas.

Estos métodos no proporcionan medidas fundamentadas en la voluntad de pagar por un producto o servicio. Por el contrario, se basan en el supuesto de que si las personas incurren en gastos para evitar daños causados por servicios de los ecosistemas perdidos o para reemplazar algún servicio de los ecosistemas, estos servicios deberían ser reemplazados. Es decir, que los métodos se aplican más correctamente en aquellas situaciones en que los gastos para evitar daños o reemplazo de factores, van a ser efectivamente realizados. Un ejemplo es el trabajo de Ramírez y Ríos $^{10}$, que presenta un modelo de valoración de costos ambientales sobre la calidad del agua potable en municipios del Departamento de Risaralda (Colombia). Por medio de este trabaj 0, se pudo establecer que la contaminación del agua por coliformes fecales era una variable significativa, aunque no la única, para explicar la morbilidad por enfermedad diarreica aguda (EDA). Se comprobó además que las deficientes condiciones de tratamiento y desinfección afectaban la salud de los pobladores de los municipios del departamento, especialmente en lo que se refiere a la población con necesidades básicas insatisfechas o la población más vulnerable.

El método de los precios hedónicos se basa en la teoría de las características ${ }^{11}$, y consiste en identificar la influencia de la calidad ambiental sobre determinados factores sobre el valor final de un bien. Es decir, que el método permite explicar el valor total de un bien en función de un conjunto de características. Por ejemplo, una planta de regeneración sería preferida en función de variables como la velocidad, eficiencia energética o dimensión (entre otras). De lo anterior, el valor total de un bien dependerá de la evaluación de todas sus características y, como en cualquier función de demanda, el cambio en una de las características puede modificar el precio final del bien.

Una de las principales limitaciones de este método reside en la gran necesidad de información estadística para poder identificar todas las variables explicativas. La fiabilidad de los datos

\footnotetext{
9 ECM, 2005.

10 Ramírez y Ríos, 2010

11 Esta teoría fue inicialmente propuesta por Lancaster, 1971, y desarrollada posteriormente por Griliches, 1971, y Rosen, 1974.
}

es otra de las principales restricciones de este método, así como los valores de no-uso del bien, o las posibles expectativas de los consumidores sobre la evolución de los precios.

Debido a la relación entre bien privado y bien ambiental que fundamenta los métodos de preferencias reveladas, la aplicación de estos métodos se circunscribe normalmente a la estimación de los valores de uso. Otra limitación de los métodos de preferencias reveladas es que solo pueden medir la valoración de los bienes a posteriori, una vez consumidos, por lo que no permiten la estimación de valores para niveles de calidad que todavía no han sido experimentados.

En resumen, los métodos de preferencias reveladas se basan en las relaciones que se establecen entre los bienes o servicios ambientales valorados y los que se adquieren en el mercado. Una hipótesis fundamental es que las personas revelan en su comportamiento con respecto al bien privado el valor que realmente se le otorga al bien ambiental.

\section{Métodos de preferencias declaradas}

Los métodos de preferencias declaradas se refieren a un conjunto de técnicas basadas en las declaraciones de individuos sobre sus preferencias al plantearse cuáles son sus preferencias entre diversas opciones que describen un conjunto de situaciones 0 escenarios hipotéticos. Esta es la principal diferencia con los métodos de preferencias reveladas, que se fundamentan sobre situaciones observadas.

Los métodos de preferencias declaradas necesitan del diseño de encuestas específicas para obtener la información necesaria. Aquí aparece un primer aspecto fundamental: definir las variables relevantes que intervienen en el modelo de valoración, así como los niveles en que estas variables determinan los resultados. La siguiente etapa en la construcción del diseño es analizar las respuestas obtenidas. En términos generales, se pueden distinguir tres tipos de respuestas declaradas:

- Elección. El encuestado selecciona en cada escenario planteado la alternativa preferida.

- Jerarquización. El encuestado ordena las alternativas presentadas de acuerdo a sus preferencias.

- Escalamiento. El encuestado presenta el grado de preferencia entre opciones a partir de una escala semántica. Es decir, que se presentan unas posibilidades de respuesta ordenables y semánticamente autónomas y con un significado acabado (por ejemplo, buenísimo, bueno, normal, menos bueno).

Es importante destacar que para garantizar la calidad de la encuesta suelen realizarse encuestas piloto o trabajos simulados antes de su realización.

El Método de Valoración Contingente es uno de los métodos de preferencias declaradas más representativo. Este método simula un mercado mediante un cuestionario, en el que el encuestador realiza el papel de oferente del bien y el encuestado de demandante ${ }^{12}$. El cuestionario establece las condiciones en las que

12 Mitchell \& Carson, 1989 
tendría lugar el intercambio y por ello se conoce como escenario de la valoración, simulando un cambio en la provisión del bien. Entonces, se pregunta al individuo por la máxima cantidad de dinero que estaría dispuesto a pagar 0 , alternativamente, se le presenta un precio que la persona entrevistada puede aceptar o no.

Los principales métodos de elección son dos: el método de ordenación contingente y los experimentos de elección. La diferencia sustancial radica en que, mientras que en el experimento de elección se solicita a las personas entrevistadas la elección de la opción preferida entre diferentes alternativas, la ordenación contingente se fundamenta en la ordenación de las alternativas según sus propias preferencias. Las alternativas están compuestas por diferentes combinaciones de bienes (que se describen mediante sus atributos) y el coste asociado a cada una de ellas. Estos métodos presentan la ventaj a respecto a los basados en la valoración contingente de que obligan a los electores a representar sus métodos de elección entre un conj unto de atributos, por ejemplo en el caso de unos humedales ${ }^{13}$.

Los métodos de los Experimentos de Elección consisten en presentar a la persona entrevistada un conjunto de alternativas que contienen atributos comunes de un bien, pero con diferentes características. Al sujeto entrevistado se le solicita que elija la alternativa preferida para cada conj unto. Cada conjunto de elección se realiza entre una alternativa constante (el estado actual en el cual se encuentra el bien sin ningún cambio) y una serie de alternativas propuestas. La elección realizada por el individuo indica una preferencia por los atributos de una alternativa respecto de las otras. Este método se fundamenta en valorar cambios en los atributos del bien, lo que permite transformar las respuestas en una estimación y en magnitudes monetarias.

En cuanto a unos humedales artificiales en los que se utiliza agua regenerada, los métodos de elección podrían utilizarse si, en lugar de valorar un escenario determinado, pudiera interesar la valoración separada de los distintos indicadores 0 atributos del humedal. Estos podrían ser, por ejemplo, el grado de biodiversidad, la calidad del suelo, problemas relacionados con la intrusión salina, o algunos elementos específicos del propio paisaje de humedales. En una situación como esta, el ayuntamiento de la zona afectada podría estar interesado en conocer el valor de los distintos atributos de la zona, como podrían ser la presencia de determinadas aves migratorias, el valor de las actividades recreativas o la posibilidad de desarrollar actividades productivas relacionadas con la agricultura.

En estos casos, la valoración ambiental de un humedal construido también podría fundamentarse en la aplicación reiterada del método de valoración contingente, si bien en algunos casos podría ser más precisa la aplicación de otros métodos alternativos que permitieran estimar los diferentes valores por separado. De este modo podría recogerse la valoración de atributos diversos, así como la consideración de diferentes medidas para representar la calidad o cantidad del agua residual utilizada en el humedal.

La forma de plantear las preguntas resulta fundamental en la aplicación del método y constituye una característica esencial del procedimiento utilizado. Existen diversas variantes:

13 Birol et al. 2006 .
- Si a la persona entrevistada se le pide que ordene un conjunto de alternativas según sus preferencias, este método se conoce como ordenación contingente (contingent ranking).

- Si se le solicita que puntúe cada una de las alternativas en una escala (por ej emplo, entre 1 y 10), este método se conoce como contingent rating.

- Si se le requiere que elija la opción preferida entre un conjunto de alternativas, entonces se denomina método de los experimentos de elección (choice experiment).

\section{Conclusiones}

El análisis económico y financiero de los proyectos relacionados con los sistemas de regeneración y reutilización de aguas residuales se ha concentrado tradicionalmente en los costes y beneficios privados, tanto por las dificultades metodológicas relacionadas con la estimación de las externalidades existentes, como por la disponibilidad de los datos necesarios para su valoración.

La consideración de los costes y beneficios permite una mejora sustancial en el diseño y gestión de los sistemas de regeneración y reutilización de aguas residuales, ambientales, tanto públicos como privados. Como consecuencia, también se origina una mejora en la gestión de las inversiones en estos sistemas de tratamiento.

La reutilización de aguas residuales es una actividad con tendencia al monopolio natural, a causa de los muy elevados costes relacionados con la instalación de las infraestructuras necesarias para el tratamiento, distribución y gestión del agua. No suele ser rentable, desde el punto de vista económico privado, la oferta de estos servicios cuando existe más de una empresa en un mercado.

La posición monopolística, combinada con la necesidad básica del agua convierte la provisión de agua reutilizada en un servicio muy susceptible al entorno económico y político, tanto en su creación, como en su expansión en el largo plazo.

\section{Bibliografía}

Birol, E., Karousakis, K., y Koundouri, P. 2006: "Using a choice experiment to account for preference heterogeneity in wetland attributes: The case of Cheimaditida wetland in Greece", en Ecological Economics, 60, 1, 145-156. http://dx. doi. org/10.1016/j. ecolecon. 2006.06.002.

Drechsel, P. y Evans, A. E. V. 2010 "Wastewater Use in Irrigated Agriculture", en Irrigation and Drainage Systems, 24, 1, 1-3. doi:10.1007/ s10795-010-9095-5

Drechsel, P., Scott, CA, Raschid-Sally, L., Redwood, M., B. y Bahri, A. 2010: "Wastewater Use in Agriculture: Challenges in Assessing Costs and Benefits", en Drechsel, P., Qadir, M. y Wichelns, D. (edits.): Wastewater. Colombo, Springer, 139-152.

Ensink, J. H. y Van der Hoek, W. 2009: "Implementation of the WHO guidelines for the safe use of wastewater in Pakistan: balancing risks and benefits", en J ournal of Water and Health, 7, 3, 464-468. doi: 10.2166/ wh. 2009.061. PMid: 19491496

Evaluación de los Ecosistemas del Milenio, 2005: Evaluación de los Ecosistemas del Milenio. Washington, D.C., Island Press. 
Griliches, Z. 1971: Price Indexes and Quality Change. Studies in New Methods of Measurement. Cambridge, Harvard University Press. https:// doi. org/ 10.4159/ harvard. 9780674592582.

Hamilton, A. J., Stagnitti, F., Xiong, X., Kreidl, S. L., Benke, K. K. y Maher, P. 2007: "Wastewater Irrigation: The State of Play", en Vadose Zone J ournal, 6, 4, 823-840. doi:10.2136/ vzj2007.0026.

Hurlimann, A. y McKay, J. 2007: "Urban Australians using recycled water for domestic non-potable use. An evaluation of the attributes price, saltiness, colour and odour using conjoint analysis", en J ournal of Environmental Management, 83, 1, 93-104. http://dx.doi. org/10.1016/j.jenvman. 2006.02.008. PMid: 16678338

Jiménez, B. y Asano, T. 2008: "Water reclamation and reuse around the world", en Jiménez B. y Asano, T. (coords.): Water Reuse: An International Survey of Current Practice, Issues and Needs. Londres, IWA, 1-26. DOI: 10.2166/9781780401881

Keraita, B., Drechsel, P. y Konradsen, F. 2010: "Up and down the sanitation ladder: Harmonizing the treatment and multiple-barrier perspectives on risk reduction in wastewater irrigated agriculture", en Irrigation and Drainage Systems, 24, 1, 23-35. DOI 10.1007/ s10795-009-9087-5.

Lancaster, K. 1971: Consumer demand: A new approach. New York, Columbia University Press. PMCid: PMC197617

Mills, R. A., Karajeh, F. y Hultquist, R. H. 2004: "California's Task Force evaluation of issues confronting water reuse", en Water Science and Technology, 50, 2, 301-308. PMid: 15344805

Mitchell, R. C. y Carson, R. T. 1989: Using surveys to value public goods: The contingent valuation method. Washington, Resources for the Future.

Molinos-Senante, M., Garrido-Baserba, M., Reif, R., Hernández-Sancho, F. y Poch, M. 2012: "Assessment of wastewater treatment plant design for small communities: Environmental and economic aspects", en Science of The Total Environment, 427-428, 11-18. http:// dx. doi. org/ 10.1016/ j. scitotenv. 2012.04.023. PMid: 22578695

Pescod, M. B. 1992: "The Urban Water Cycle, including Wastewater Use in Agriculture", en Outlook on Agriculture, 21, 4, 263-270.

Qadir, M., Sharma, B. R, Bruggeman, A., Choukr-Allah, R., Karajeh, F. 2007a: "Non-conventional water resources and opportunities for water augmentation to achieve food security in water scarce countries", en Agricultural Water Management, 87, 1, 2-22. http:// dx. doi. org/ 10.1016/ j. agwat. 2006. 03.018.

Qadir, M., Wichelns, D., Raschid-Sally, L., Minhas, P. S., Drechsel, P., Bahri, A. y McCornick, P. 2007b: "Agricultural use of marginal-quality water- opportunies and challenges", en Molden, D. : Water for food. Water for life: A Comprehensive Assessment of Water Management in Agricultura. London, Earthscan, 425-457.

Qadir, M., Scott., C. A. 2010: "Non-pathogenic trade-offs of wastewater irrigation", en Drechsel, P., Scott, C. A. , Raschid-Sally, L., Redwood, M. y Bahri, A. (edits.): Wastewater irrigation and health: assessing and mitigating risks in low-income countries. London, EarthscanInternational Development Research Centre (IDRC)-International Water Management Institute (IWMI), 101-126.

Qadir, M., Mateo-Sagasta, J., Jiménez, B., Siebe, C., Siemens, J. y Hanjra, M. A. 2015: "Environmental Risks and Cost-Effective Risk Management in Wastewater Use Systems", en Drechsel, P., Qadir, M. y Wichelns, D. (eds.): Wastewater. Colombo, Springer, 55-72. https:// doi. org. 10.1007/ 978-94-017-9545-6-4

Raschid-Sally, L. y Jayakody, P. 2008: Drivers and Characteristics of Wastewater Agricultura in Developing Countries: Results from a Global Assessment. Research Report 127. Colombo, Sri Lanka, International Water Management Institute.

Ramírez Vasco, C. L., Ríos Millán, C. P. y Morales Pinzón, T. 2010. “Estimación de costos inducidos derivados de la calidad del agua potable en Risaralda", en Scientia et Technica, 44, 117-122.

Rosen, S. 1974: "Hedonic Prices and Implicit Markets: Product Differentiation in Pure Competition", en J ournal of Political Economy, 82, 1, 34-55. https:// doi. org/ 10.1086/ 260169

Scheierling, S. M., Bartone, C. R., Duncan Mara, D., Drechsel, P. 2011: "Towards an agenda for improving wastewater use in agriculture", en Water International, 36, 4, 420-440. http:// dx. doi.org/ 10.1080 / 02508060. 2011.594527.

Scott, C. A., Faruqui, N. I. y Raschid-Sally, L. 2004: Wastewater Use in Irrigated Agriculture. Wallingford, CAB International-IWMI-IDRC.

Scott, C. A., Drechsel, P., Raschid-Sally, L., Redwood, M., y Bahri, A. (edits.) 2010: Wastewater Irrigation and Health: Assessing and Mitigating Risk in Low-Income Countries. London, Earthscan-International Development Research Centre-International Water Management Institute.

Siemens, J., Huschek, G., Siebe, C., Kaupenjohann, M. 2008: "Concentrations and mobility of human pharmaceuticals in the world's largest wastewater irrigation system, Mexico City-Mezquital Valley", en Water Research, 42, 8-9, 2124-2134. http:// dx. doi.org/10.1016/ j. watres.2007.11.019. PMid: 18083208

Simmons, R., Qadir, M. y Drechsel, P. 2010: "Farm-based measures for reducing human and environmental health risks from chemical constituents in wastewater" en Drechsel, P., Scott, C. A., Raschid-Sally, L., Redwood, M. y Bahri, A. (edts.): Wastewater irrigation and health: assessing and mitigating risk in low-income countries. London, UK: Earthscan; Ottawa, Canada: International Development Research Centre (IDRC); Colombo, Sri Lanka: International Water Management Institute (IWMI), 209-238.

Van Roon, M. 2007: "Water localization and reclamation: steps towards low impact urban design and development", en J ournal of Environmental Management, 83, 4, 437-447.

World Health Organization (WHO), 2006: "Wastewater use in agricultura", en Guidelines for the safe use of wastewater, excreta and greywater (Vol. 2), en http:// www. who. int/ water_sanitation_health/ wastewater/ wwuvol2intro. pdf. 